\title{
Dexamethasone Inflammatory Suppression F-18-FDG PET/CT: Restaging Pitfall in a Patient with Synchronous Laryngeal and Pulmonary Carcinomas: A Case Report
}

Tiago S Vieira ${ }^{1^{*}}$, Diogo B Faria ${ }^{2}$, Fernando A Silva ${ }^{1}$, Daniel P Coutinho ${ }^{3}$, Francisco Pimentel ${ }^{1}$ and José Oliveira ${ }^{1}$

${ }^{1}$ HPP-Medicina Molecular SA, Lenitudes Medical Center and Research, Porto, Portugal

${ }^{2}$ HPP-Medicina Molecular SA, Lenitudes Medical Center and Research, School of Health Sciences - University of Aveiro, Portugal

${ }^{3}$ Centro Hospitalar de Vila Nova de Gaia/Espinho, Portugal

*Corresponding author: Tiago S Vieira, HPP-Medicina Molecular SA, Lenitudes Medical Center and Research, Apartado 1064, EC Pedro Hispano 4101-001 Porto, Portugal, E-mail: tiago.sampaio.vieira@gmail.com

Received date: September 18, 2018; Accepted date: October 04, 2018; Published date: October 11, 2018

Copyright: @2018 Vieira TS, et al. This is an open-access article distributed under the terms of the Creative Commons Attribution License, which permits unrestricted use, distribution, and reproduction in any medium, provided the original author and source are credited.

\begin{abstract}
:
Rationale: F-18-FDG PET/CT is used in a wide range of oncological settings and has high accuracy to detect second primary tumors in patients with head and neck cancers. Some factors, which need to be recognized, may affect F-18-FDG PET interpretation.

Patient Concerns: We present the case of a 69-year-old male who performed F-18-FDG PET/CT for staging of a laryngeal squamous cell carcinoma.

Diagnoses: Staging PET/CT revealed hypermetabolism in the laryngeal cancer and identified a second primary pulmonary squamous cell carcinoma and subcarinal lymph node metastases. The patient refused treatment and was lost to follow-up, returning to the Hospital 4 months later due to upper airway obstruction. Restaging F-18-FDG $\mathrm{PET} / \mathrm{CT}$ revealed less intense uptake than previously verified at both the primary tumors and the metastases, and a thorough analysis of the clinical process revealed that the patient had been under dexamethasone for 7 days prior to the restaging PET.

Interventions: After the restaging PET the patient completed 7 cycles of chemotherapy, which he then decided to suspend.

Outcomes: Dexamethasone inflammatory suppression was identified as the causative factor for the apparent metabolic improvement of the malignant lesions on the restaging PET. The patient died 10 months after chemotherapy interruption due to progression.

Lessons: F-18-FDG PET/CT is a useful tool for the identification of second primary tumors in patients with head and neck cancers. This case report highlights the importance of searching for ongoing corticotherapy when performing F-18-FDG PET, to avoid wrong clinical interpretations of imaging, especially during follow-up.
\end{abstract}

Keywords: Dexamethasone suppression F-18-FDG PET/CT; Synchronous laryngeal and pulmonary squamous cell carcinomas; Restaging pitfall

Abbreviations: F-18-FDG PET/CT: F18-Fluorodeoxyglucose Positron Emission Tomography/Computed Tomography; SUV $\max$ : Maximum Standardized Uptake Value

\section{Introduction}

F-18-FDG PET/CT imaging is now used in a wide range of oncological settings, although F-18-FDG is not a tumor-specific imaging agent and inflammatory cells are also F-18-FDG avid [1,2].

Up to $18 \%$ of head and neck cancer patients have a second primary malignancy [3], especially when the index tumor is a laryngeal carcinoma, and most are found in the lung [4]. PET/CT has high accuracy for the detection of second primary cancers in patients with head and neck cancers [5].

PET is also useful for therapy response assessment of oncological patients, but care must be taken to control the factors that may affect

F-18-FDG biodistribution, namely ongoing glucocorticoid therapy and its effect on blood glucose levels [6,7].

On the other hand, the cellular inflammatory infiltration contributes to the amount of F-18-FDG uptake quantified in tumors $[8,9]$ and here we report the rare finding of a restaging pitfall due to dexamethasone inflammatory suppression while performing F-18FDG PET/CT in a patient with synchronous laryngeal and pulmonary carcinomas. 
Citation: Vieira TS, Faria DB, Silva FA, Coutinho DP, Pimentel F, et al. (2018) Dexamethasone Inflammatory Suppression F-18-FDG PET/CT: Restaging Pitfall in a Patient with Synchronous Laryngeal and Pulmonary Carcinomas: A Case Report. J Nucl Med Radiat Ther 9: 381. doi:10.4172/2155-9619.1000381

Page 2 of 3

\section{Case Report}

We present the case of a 69 years-old male with squamous cell carcinoma of the larynx who underwent a staging F-18-FDG PET/CT. This staging PET confirmed hypermetabolism in the laryngeal tumor $\left(\mathrm{SUV}_{\max }=9.7\right)$ and revealed another focus of increased uptake in the right lung $\left(\mathrm{SUV}_{\max }=11.2\right)$, which was later characterized as a second primary squamous cell carcinoma of the lung, as well as subcarinal lymph node metastases $\left(\mathrm{SUV}_{\max }=3.5\right)$ (Figure 1).

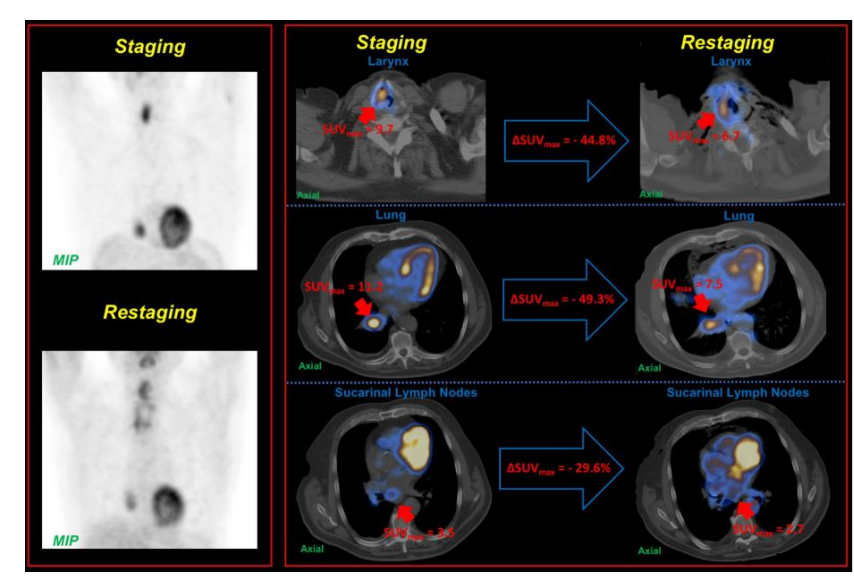

Figure 1: Staging F-18-FDG PET/CT axial images confirming hypermetabolism in the known laryngeal tumor and showing foci of increased uptake in a right lung second primary tumor and in subcarinal lymph node metastases (images on the left side). Restaging F-18-FDG PET/CT revealed less intense uptake in the malignant lesions and no evidence of new foci of increased uptake suggestive of malignancy (images on the right side).

The patient refused anticancer therapy and was lost to follow-up, returning to the hospital 4 months later due to breathing difficulties resulting from upper airway obstruction which determined a tracheostomy.

A restaging F-18-FDG PET/CT revealed less intense uptake than previously found in both the laryngeal and pulmonary primary tumors $\left(\Delta \mathrm{SUV}_{\max }=-44.8 \%\right.$ and $-49.3 \%$, respectively) and the metastases $\left(\Delta S U V_{\max }=-29.6 \%\right)$, and there was no evidence of new foci of increased uptake suggestive of malignancy (Figure 1).

A thorough analysis of the clinical process revealed that the patient had been under intravenous dexamethasone therapy $(8 \mathrm{mg} /$ day $)$ for 7 days prior to the restaging PET, and this fact was interpreted as the probable cause for the decrease observed on the metabolic activity of the malignant lesions.

After the restaging PET the patient completed 7 cycles of palliative chemotherapy with carboplatin and paclitaxel during approximately four and half months. He decided to suspend the anticancer medication and died ten months later due to disease progression.

\section{Discussion}

Showing hypermetabolism in the known laryngeal tumor, the staging F-18-FDG PET performed on this patient allowed the identification of an unknown synchronous lung tumor. Indeed, up to $18 \%$ of the patients with head and neck cancers have a second primary malignancy [3], especially when the index tumor is a laryngeal carcinoma, and the majority are found in the lung [4], being more frequently a squamous cell carcinomas [10], as in this case. The fact that F-18-FDG PET/CT was able to identify an unknown tumor of the lung is in line with the high accuracy reported regarding this functional imaging technique for the detection of second primary cancers in patients with head and neck cancers [5].

F-18-FDG PET is indicated for initial staging and follow-up of oncology patients, but F-18-FDG is not a tumor-specific tracer and increased uptake also occurs in inflammatory cells [1].

Standardized uptake value (SUV) is the most commonly used semiquantitative parameter for analysis of radiotracer uptake in a tumor; nevertheless, a variety of biologic and technologic factors can influence PET SUV measurements [11].

For instance, glucocorticoids can elevate serum glucose levels, which affects quantification of F-18-FDG uptake [6,7]. The patient in this case had normal and similar glucose levels during the staging and restaging PET scans and, therefore, that couldn't be the explanation for the SUV decrease found between the staging and restaging exams. Technologic factors such as F-18-FDG injected dose, timing of imaging acquisition after radiotracer administration and image reconstruction parameters were also checked and couldn't affect SUV quantification in this case.

From another perspective, F-18-FDG accumulates in both neoplastic cells and activated inflammatory cells [1], but little is known about the impact of inflammation on SUV quantification in cancer lesions. Some authors used steroidal anti-inflammatory drugs to study this subject. Honda and colleagues injected hydrocortisone $(2 \mathrm{mg} / \mathrm{kg}$ ) at 48,24 , and 6 hours before intravenous administration of F-18-FDG in rats with implanted tumors and no significant uptake variations were noted at any time [12]. In its turn, Brepoels et al also inoculated mice with tumors, which were then treated with hydrocortisone $(0.2$ $\mathrm{mg}$ /day) for 5 days, and the contribution of inflammatory cells to F-18FDG uptake on tumors after corticosteroid administration proved to be less important than when chemotherapy was used alone [8], showing that nonspecific F-18-FDG uptake affects the analysis of changes in the amount of viable tumor cells. Moreover, recently Nakajo et al. evaluated the value of dexamethasone suppression F-18-FDG PET (8 mg dexamethasone was administered orally 24 hours before F-18-FDG injection) for differentiating between true-positive and false-positive lesions in humans with non-small cell lung cancer, reporting a significantly lower $\mathrm{SUV}_{\max }$ in primary tumors on dexamethasone suppression than on baseline scans [9].

This way, although there are still few studies using corticosteroids to evaluate the influence of nonspecific F-18-FDG uptake in inflammatory cells on the quantification of the amount of viable tumor cells in cancer lesions, the research available seems to support an impact of inflammation, and differences in results may be related to the type, timing and dosage of corticosteroid administered before PET performance.

In this case the technological and biological factors known to affect SUV measurement of F-18-FDG were excluded by technical a clinical analysis and, since the patient didn't receive chemotherapy or any other anticancer treatment after the staging PET, the apparent restaging decrease on the amount of viable tumor cells was interpreted as a pitfall related to dexamethasone suppression of nonspecific F-18FDG uptake in activated inflammatory cells present in the primary and metastatic cancer lesions. 
Citation: Vieira TS, Faria DB, Silva FA, Coutinho DP, Pimentel F, et al. (2018) Dexamethasone Inflammatory Suppression F-18-FDG PET/CT: Restaging Pitfall in a Patient with Synchronous Laryngeal and Pulmonary Carcinomas: A Case Report. J Nucl Med Radiat Ther 9: 381. doi:10.4172/2155-9619.1000381

Page 3 of 3

This case underlines the value of F-18-FDG PET/CT for the detection of second primary cancers in patients with laryngeal carcinomas. Moreover, using the rare finding of a dexamethasone inflammatory suppression restaging pitfall in a clinical setting it is highlighted the crucial importance of always looking for the possibility of ongoing corticotherapy when performing F-18-FDG PET/CT, to avoid wrong clinical interpretations of imaging, especially during the follow-up of cancer disease patients.

There is still limited knowledge about the impact of activated inflammatory cells on the analysis of viable tumoral cells in cancers while performing F-18-FDG PET/CT and the importance of the subject makes urgent the need for more research addressing this issue.

\section{Conflicts of Interest}

The authors have no funding or conflicts of interest to disclose.

\section{Ethical Approval}

This study was approved by the Lenitudes Saúde Ethics Committee.

\section{References}

1. Boellaard R, Delgado-Bolton R, Oyen WJG (2015) FDG PET/CT: EANM procedure guidelines for tumor imaging. version 2.0. Eur J Nucl Med Mol Imaging 42: 328-354.

2. Fletcher JW, Djulbegovic B, Soares HP (2008) Recommendations on the use of 18F-FDG PET in oncology. J Nucl Med 49: 480-508.

3. Kim SY, Roh JL, Yeo NK (2007) Combined 18F-fluorodeoxyglucosepositron emission tomography and computed tomography as a primary screening method for detecting second primary cancers and distant metastases in patients with head and neck cancer. Ann Oncol 18: 1698-1703.

4. Morris LGT, Sikora AG, Hayes RB, Patel SG, Ganly I (2011) Anatomic sites at elevated risk of second primary cancer after an index head and neck cancer. Cancer Causes Control 22: 671-679.

5. Xu G-Z, Guan D-J, He Z-Y (2011) 18FDG-PET/CT for detecting distant metastases and second primary cancers in patients with head and neck cancer. A meta-analysis. Oral Oncol 47: 560-565.

6. Surasi DS, Bhambhvani P, Baldwin JA, Almodovar SE, O’Malley JP (2014) 18F-FDG PET and PET/CT patient preparation: A review of the literature. J Nucl Med Technol 4: 5-13.

7. (2016) ACR-SPR practice parameter for performing FDG-PET/CT in oncology, American College of Radiology.

8. Brepoels L, Stroobants S, Vandenberghe P (2007) Effect of Corticosteroids on 18F-FDG Uptake in Tumor Lesions After Chemotherapy. J Nucl Med 48: 390-397.

9. Nakajo M, Nakajo M, Nakayama H (2016) Dexamethasone suppression FDG PET/CT for differentiating between true- and false-positive pulmonary and mediastinal lymph node metastases in non-small cell lung cancer: A pilot study of FDG PET/CT after oral administration of dexamethasone. Radiology 279: 246-253.

10. Jones AS, Morar P, Phillips DE, Field JK, Husband D, et al. (1995) Second primary tumors in patients with head and neck squamous cell carcinoma. Cancer 75: 1343-1353.

11. Adams MC, Turkington TG, Wilson JM, Wong TZ (2010) A systematic review of the factors affecting accuracy of SUV measurements. AJR Am J Roentgenol 195: 310-320.

12. Honda G, Suzuki M, Yamaguchi K, Ito M (2003) Steroid reduces the 18 FDG accumulation to the chronic inflammatory foci. J Nuc Med 44 : $78 \mathrm{P}$. 